\title{
Sách vẫn còn giá trị sử dụng
}

\author{
Hồ Mạnh Toàn \\ Trường Đại học Phenikaa
}

Kinh tế và Dự báo

Hà Nội, Ngày 19 tháng 3 năm 2021

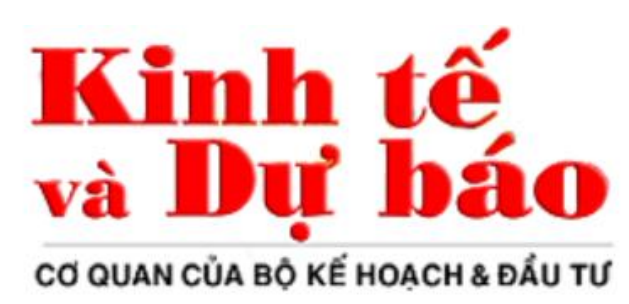

http://kinhtevadubao.vn/chi-tiet/230-18346-sach-van-con-gia-tri-su-dung.htm/ 


\section{Sách vẫn còn giá trị sử dụng}

Thời đại hiện nay, trong giới học thuật, các bài báo trên các tạp chí 'peer-review' có một vai trò không những quan trọng, mà còn quyết định uy tín và tương lai các nhà khoa học. Các nhà nghiên cứu bỏ rất nhiêu công sức và tâm huyết vào việc tiến hành nghiên cứu khoa học, và thường trực mối quan tâm xuất bản bài tạp chí [1]. Thành công trong xuất bản các bài báo khoa học có giá trị cao cũng mang lại rất nhiều lợi thế cho người làm nghiên cứu trong suốt cả sự nghiệp.

Tuy vậy, sách học thuật vẫn có chỗ đứng của chúng trong kho tàng khoa học nhân loại, mặc dù không còn vị thế trọng yếu như thủa xa xưa.

Trong khuôn khổ dự án nghiên cứu SSHPA của AISDL (được trình bày chi tiết trong tài liệu đã công bố [2]), cuốn sách [3] được xuất bản bởi De Gruyter (Imprint: Sciendo) năm 2019.

Mọi người đều mong chờ xem số phận của cuốn sách được đón nhận ra sao.

Cuốn sách xuất bản đầu tháng 11-2019, sau đó khoảng 6 tuần, nó đã được liệt kê trong tàng thư của Thư viện Hoàng gia Hà Lan, Koninklijke Bibliotheek (Hình 1).

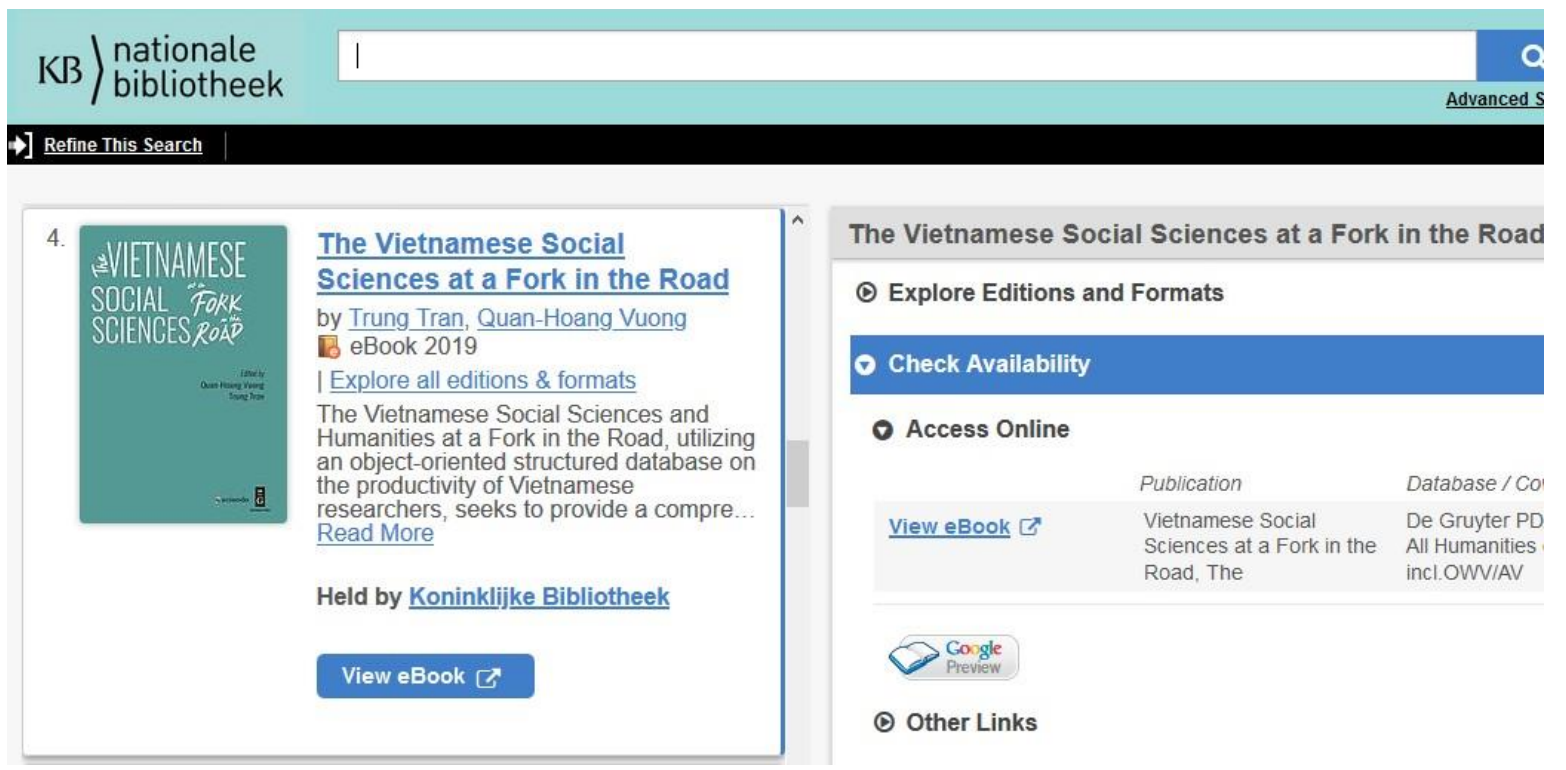

Hinh 1. Koninklijke Bibliotheek; https://www.kb.nl/

Việc xuất hiện trong Koninklijke Bibliotheek là một sự vinh hạnh, cũng là dấu hiệu khả quan. Hà Lan là quốc gia có trình độ học vấn rất cao, và nền khoa học phát triển sớm hàng đầu thế giới. Và điều này cũng có ngụ ý, văn hóa đọc rất phát triển.

Quả nhiên, gần như đồng thời, cuốn sách kể trên cũng xuất hiện ở các thư viện đại học danh tiếng của Hà Lan như Thư viện Đại học Groningen, Thư viện Đại học Tilburg. 


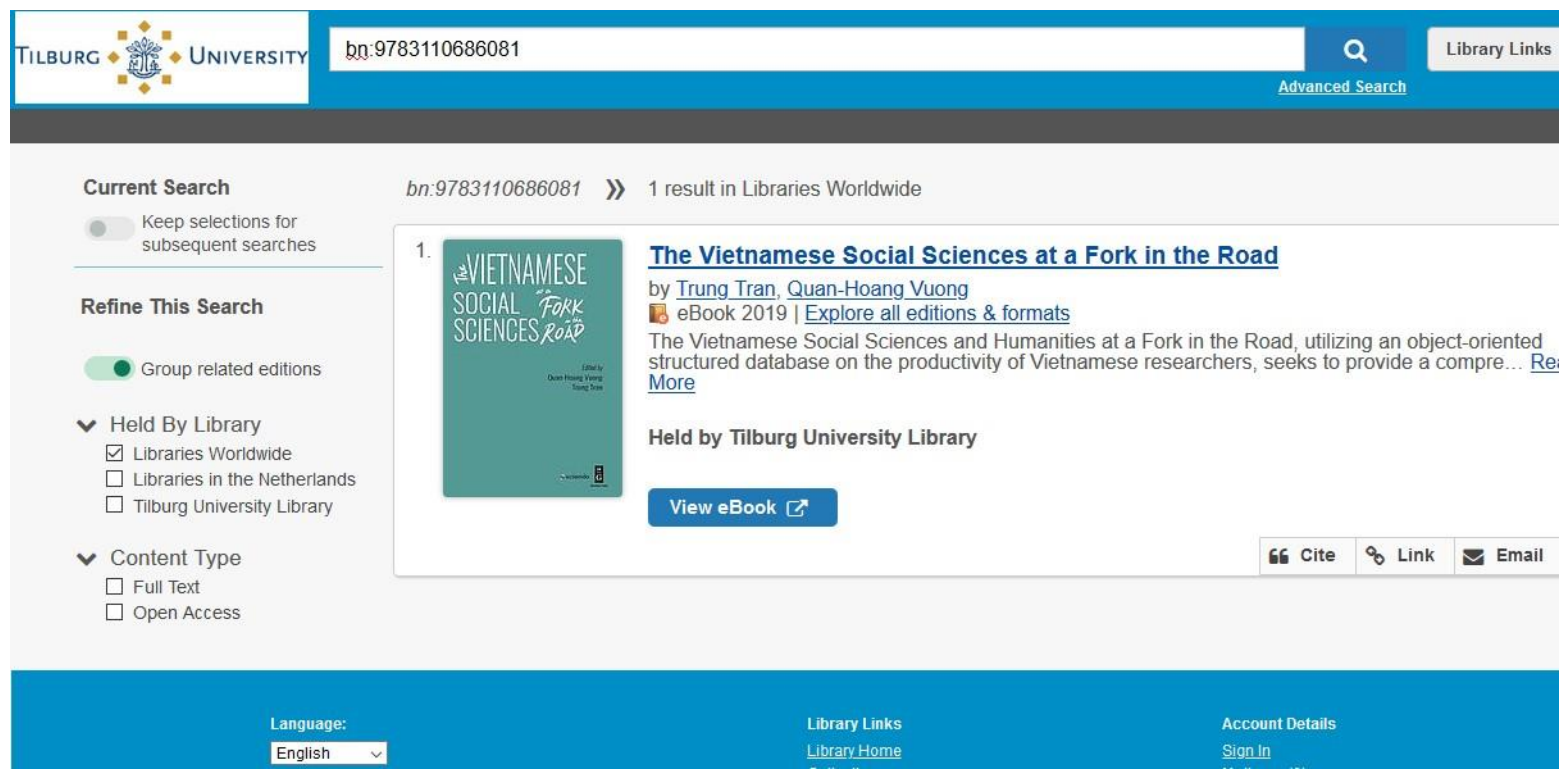

Hinh 2. Universiteit van Tilburg, Bibliotheek

Trong Hình 2 là kết quả tìm sách từ thư viện điện tử của Đại học Tilburg, Hà Lan.

Sau đó đôi tuần, [3] lại tiếp tục xuất hiện trên hệ thống lưu trữ của Thư viện Quốc gia Thụy điển LIBRIS (như trong Hình 3, ảnh chụp màn hình ngày 2-4-2020).

Với hệ thống của LIBRIS, lưu trữ của họ kết nối với nhiêu thư viện thành viên, do đó trong ảnh có một dòng ghi nhỏ ở phía dưới: Title available at 4 libraries. Điều này báo hiệu rằng sẽ có ít nhất 4 thư viện khác nữa ở Thụy Điển có lưu trữ cuốn sách [3].

Dựa trên thông tin của LIBRIS cung cấp, ta sẽ thấy được tại thời điểm truy xuất dữ liệu vừa nêu, 4 thư viện Thụy Điển lưu trữ sách là: Đại học Uppsala (https://www.uu.se/en), Đại học Stockholm (https://www.su.se/), Viện Công nghệ Hoàng gia KTH (https://www.kth.se/en), Đại học Công nghệ Chalmers (https://www.chalmers.se/). 


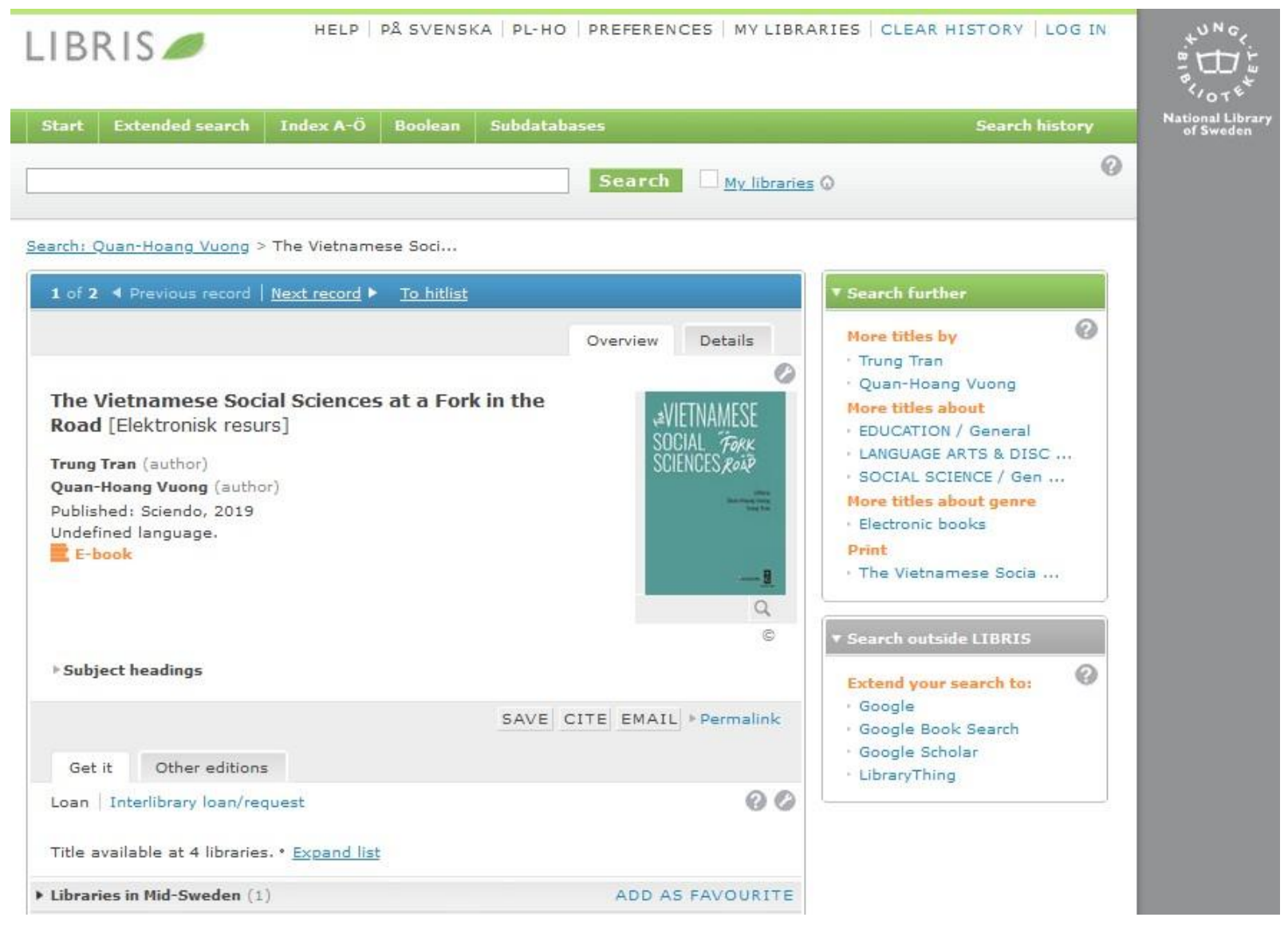

Hinh 3. National Library of Sweden; http://libris.kb.se/bib/bnbj27nx8b22tvbz.

Khi vào một trong các đường dẫn tới thư viện đại học, nếu có tài khoản truy cập thì người sử dụng (sinh viên, giảng viên) sẽ có thể tiếp cận nội dung miễn phí. Nhưng người sử dụng bắt buộc phải có UserID và mật khẩu hợp lệ.

Một ví dụ tìm thấy thông tin lưu trữ trên trang điện tử của thư viện là Học viện Công nghệ Hoàng gia $\mathrm{KTH}$, Thụy Điển trình bày trong Hình 4. Về cơ bản các thư viện đều có cùng một quy chuẩn metadata và sử dụng các cấu trúc dữ liệu dễ dàng trao đổi với nhau thông tin.

(Có một chi tiết nhỏ thú vị trong H.5: Không biết bằng cách nào mà thư viện KTH lại có thể đánh dấu tên tiếng Việt của tác giả, mặc dù trong sách không hề có dấu. Hơn nữa, họ bỏ dấu hoàn toàn chính xác, nhưng không đầy đủ!) 


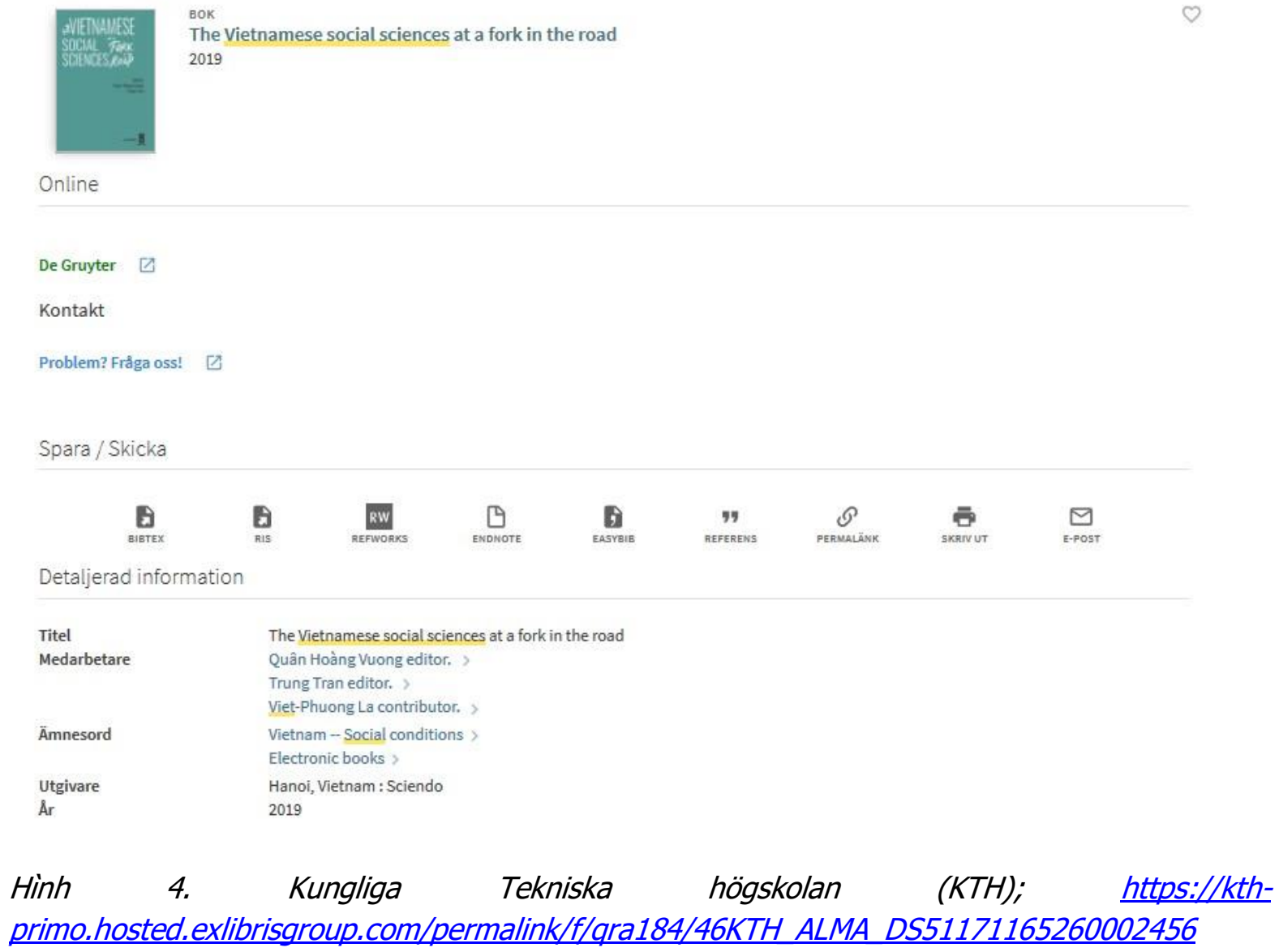

Hinh 4. Kungliga Tekniska högskolan (KTH); https://kthprimo.hosted.exlibrisgroup.com/permalink/f/qra184/46KTH ALMA DS51171165260002456

Đến đầu tháng 4-2020, lại thấy cuốn sách xuất hiện trong Thư viện Đại học New York, New York University (Hình 5).

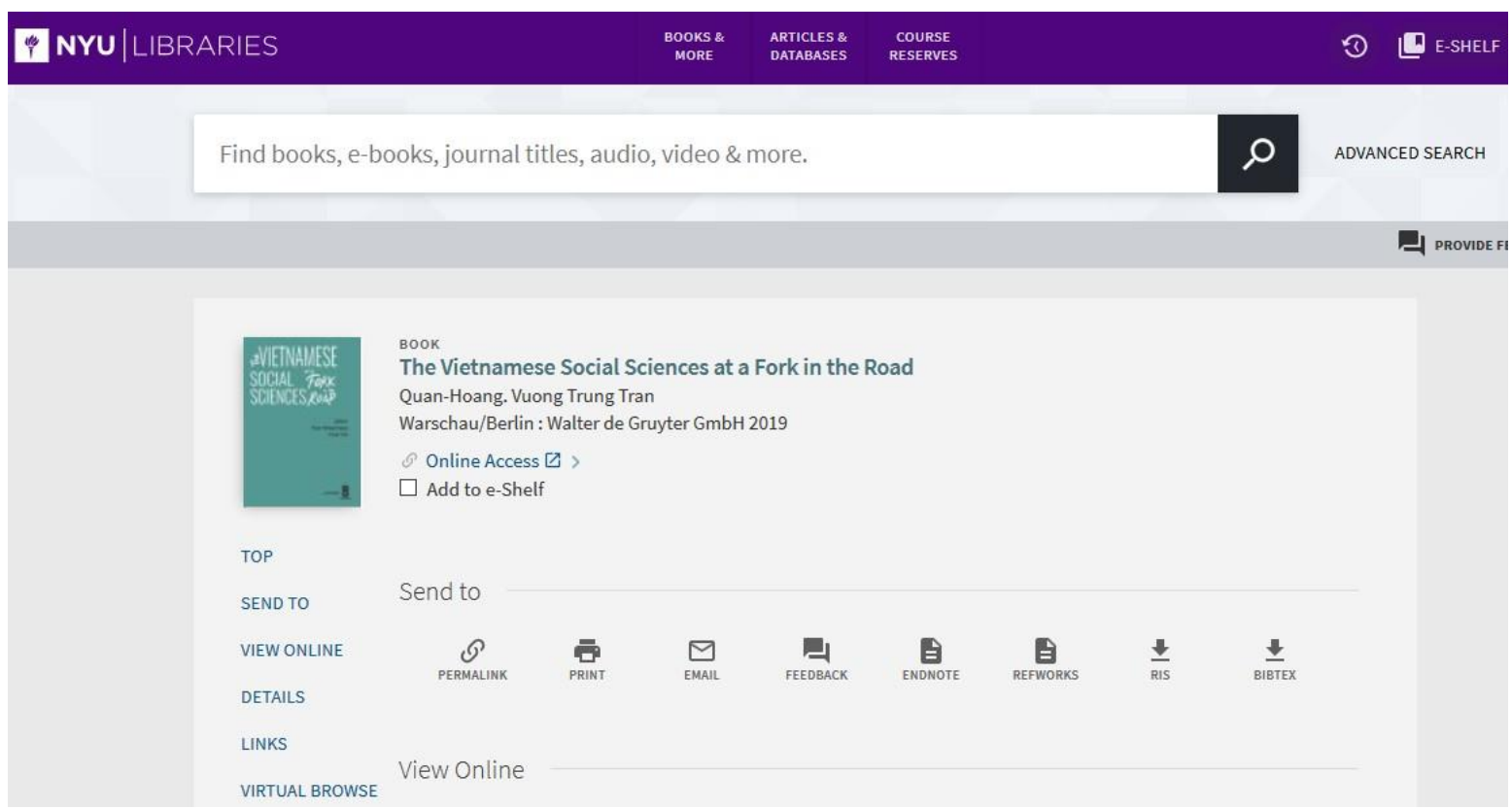


Hinh 5. Thư viện New University; https://bobcat.library.nyu.edu/permalink/f/ci13eu/nyu aleph007494635

Ngoài ra, cuốn sách cũng còn được lưu trữ tại một số thư viện đại học và cơ quan khác nữa, được liệt kê trong Bảng 1.

Bảng 1. Danh sách các thư viện lưu trữ sách [3] (tại thời điểm 2-4-2020)

\begin{tabular}{|l|l|l|}
\hline 1 & LIBRIS (National Library of Sweden). & Stockholm, S-102 41 Sweden \\
\hline 2 & New York University Library. & New York, United States \\
\hline 3 & Field Museum of Natural History. & Chicago, IL 60605 USA \\
\hline 4 & Pamukkale Üniversitesi Merkez Kütüphanesi. & Denizli, 20070 Turkey \\
\hline 5 & Uppsala universitetsbibliotek, Digitala biblioteket. & Sweden \\
\hline 6 & Rijksuniversiteit Groningen, Universiteitsbibliotheek. & $\begin{array}{l}\text { Groningen, } \\
\text { Netherlands }\end{array}$ \\
\hline 7 & Koninklijke Bibliotheek. & $\begin{array}{l}\text { Den Haag, 2595 BE } \\
\text { Netherlands }\end{array}$ \\
\hline 8 & Universiteit van Tilburg, Bibliotheek. & Tilburg, 5037 AB Netherlands \\
\hline 9 & Vancouver School of Theology Library. H.R. MacMillan Library. & $\begin{array}{l}\text { Vancouver, BC V6T 2E8 } \\
\text { Canada }\end{array}$ \\
\hline 10 & Stockholms universitetsbibliotek. & Stockholms, Sweden \\
\hline 11 & Kungliga Tekniska högskolan (KTH). & Sweden \\
\hline 12 & Chalmers tekniska högskola. & Sweden \\
\hline
\end{tabular}

( *Ghi chú: Đễ giữ kích thước bảng đủ gọn, chi tiêt thông tin và URL được đưa vào Tài liệu tham khảo, đánh số cả dãy [4-15])

Trong quá trình tìm hiểu, cũng chưa thấy thư viện ở Việt Nam có lưu trữ sách.

Sơ bộ, sau hơn 4 tháng xuất bản, một số lượng các thư viện đã lưu trữ sách để phục vụ độc giả thuộc giới nghiên cứu, phần nhiều tại các trường đại học quốc tế. Điều đó có thể phần nào giải đáp câu hỏi về giá trị sử dụng sách trong thời đại ngự trị của 'journal article'.

Bên cạnh đó, việc nhận thẫy sách nằm trong các thư viện ĐH uy tín cũng có tác dụng mang lại 'lợi tức tâm lý' (psychic return) cho lao động nghiên cứu.

\section{Tài liệu tham khảo:}

[1] Vuong QH. (2019). Breaking barriers in publishing demands a proactive attitude. Nature Human Behaviour, 3(10): 1034; doi:10.1038/s41562-019-0667-6. URL: https://www.nature.com/articles/s41562-019-0667-6. 
[2] Vuong QH, La VP, Vuong TT, ..., Ho MT. (2018). An open database of productivity in Vietnam's social sciences and humanities for public use. Scientific Data, 5: 180188. doi:10.1038/sdata.2018.188. URL: https://www.nature.com/articles/sdata2018188.

[3] Vuong QH, Tran T. (2019). The Vietnamese Social Sciences at a Fork in the Road. Warsaw, Poland: De Gruyter. doi:10.2478/9783110686081.

[4] NYU. (2020). New York University Library. New York, NY 10003, United States. https://bobcat.library.nyu.edu/permalink/f/ci13eu/nyu aleph007494635.

[5] Field Museum. (2020). Field Museum of Natural History. Chicago, IL 60605 United States: https://fieldmuseumlibrary.on.worldcat.org/oclc/1126217069.

[6] Pamukkale University. (2019). Pamukkale Üniversitesi Merkez Kütüphanesi. Denizli, 20070 Turkey. $\quad$ http://tarama.pau.edu.tr/client/tr TR/defaulttur/search/results? te $=\& \mathrm{rt}=\mathrm{OCLC} \% 7 \mathrm{C} \% 7 \mathrm{C} \% 7 \mathrm{COCLC} \% 7 \mathrm{C} \% 7 \mathrm{C} \% 7 \mathrm{Cfalse} \& q u=0 \mathrm{~cm} 1129175498$.

[7] University of Groningen Library. (2019). Rijksuniversiteit Groningen, Universiteitsbibliotheek. Groningen, 9712 CP Netherlands. https://rug.on.worldcat.org/search?queryString=bn\%3A3110686082.

[8] The Royal Library of the Netherlands. (2019). Koninklijke Bibliotheek. Den Haag, 2595 BE Netherlands. https://kb.on.worldcat.org/search?queryString=bn\%3A3110686082.

[9] Universiteit van Tilburg. (2019). Bibliotheek. Tilburg, 5037 AB Netherlands. https://tilburguniversity.on.worldcat.org/search?queryString=bn\%3A9783110686081.

[10] Vancouver School of Theology Library. H.R. MacMillan Library. Vancouver, BC V6T 2E8 Canada.

[11] LIBRIS. (2020). National Library of Sweden. Stockholm, S-102 41 Sweden. http://libris.kb.se/bib/bnbj27nx8b22tvbz.

[12] Uppsala universitetsbibliotek. (2020). Digitala biblioteket. http://ezproxy.its.uu.se/login?url=https://www.degruyter.com/openurl?genre=book\&isbn=9 $\underline{783110686081 .}$.

[13] Stockholms universitetsbibliotek. (2020). Library. http://link.sub.su.se/sfxsub?url ver=Z39.882004\&ctx ver $=Z 39.88-2004 \&$ ctx enc $=$ info:ofi/enc:UTF-

8\&rfr id=info:sid/sfxit.com:opac 856\&url ctx fmt=info:ofi/fmt:kev:mtx:ctx\&sfx.ignore date threshold=1\&rft.object id=4100000009744885\&svc val fmt=info:ofi/fmt:kev:mtx:sch svc \&.

[14] KTH Royal Institute of Technology (2020). Kungliga Tekniska högskolan. https://kthprimo.hosted.exlibrisgroup.com/permalink/f/qra184/46KTH ALMA DS51171165260002456.

[15] Chalmers University of Technology (2020). Chalmers tekniska högskola. http://search.ebscohost.com/login. aspx?direct=true\&AuthType $=$ sso\&db=edsebk\&AN=22830 $\underline{58 \& \text { site }=e d s-\text { live } \& \text { scope }=\text { site } \& \text { custid }=s 3911979 \& \text { authtype }=s \text { so\&group }=\text { main\&profile }=e d s}$. 